\title{
Chemoradiotherapy by intensity-modulated radiation therapy with simultaneous integrated boost in locally advanced or oligometastatic non-small-cell lung cancer-a two center experience
}

\author{
Frederick Mantel $^{1}$ (D) Elena Müller ${ }^{2}$ Philip Kleine ${ }^{1} \cdot$ Marcus Zimmermann $^{1} \cdot$ Florian Exner $^{1} \cdot$ Anne Richter $^{1}$. \\ Stefan Weick ${ }^{1} \cdot$ Serge Ströhle ${ }^{1} \cdot$ Bülent Polat $^{1} \cdot$ Stefan Höcht $^{2} \cdot$ Michael Flentje $^{1}$
}

Received: 8 September 2020 / Accepted: 15 February 2021 / Published online: 16 March 2021

(c) The Author(s) 2021

\begin{abstract}
Purpose Integrating moderate hypofractionation to the macroscopic tumor with elective nodal irradiation while sparing the organs at risk (OAR) in chemoradiotherapy of locally advanced non-small-cell lung cancer.

Methods From 2010-2018, treatment, patient and tumor characteristics of 138 patients from two radiation therapy centers were assessed. Chemoradiotherapy by intensity-modulated radiation therapy (IMRT) with a simultaneous integrated boost (SIB) to the primary tumor and macroscopic lymph node metastases was used.

Results A total of $124(90 \%)$ patients received concurrent chemotherapy. 106 (76\%) patients had UICC (Union for International Cancer Control) stage $\geq$ IIIB and 21 (15\%) patients had an oligometastatic disease (UICC stage IV). Median SIB and elective total dose was 61.6 and $50.4 \mathrm{~Gy}$ in 28 fractions, respectively. Furthermore, 64 patients (46\%) had an additional sequential boost to the primary tumor after the SIB-IMRT main series: median 6.6Gy in median 3 fractions. The median cumulative mean lung dose was 15.6 Gy (range 6.2-29.5 Gy). Median follow-up and radiological follow-up for all patients was 18.0 months (range 0.6-86.9) and 16.0 months (range 0.2-86.9), respectively. Actuarial local control rates at 1,2 and 3 years were $80.4,68.4$ and $57.8 \%$. Median overall survival and progression-free survival was 30.0 months (95\% confidence interval [CI] 23.5-36.4) and 12.1 months (95\% CI 8.2-16.0), respectively. Treatment-related toxicity was moderate. Radiation-induced pneumonitis grade 2 and grade 3 occurred in $13(9.8 \%)$ and $3(2.3 \%)$ patients.

Conclusions Chemoradiotherapy using SIB-IMRT showed promising local tumor control rates and acceptable toxicity in patients with locally advanced and in part oligometastatic lung cancer. The SIB concept, resulting in a relatively low mean lung dose, was associated with low numbers of clinically relevant pneumonitis. The overall survival appears promising in the presence of a majority of patients with UICC stage $\geq$ IIIB disease.
\end{abstract}

Keywords Image-guided radiation therapy $\cdot$ Thoracic cancer $\cdot$ Hypofractionation $\cdot$ Multimodal therapy $\cdot$ Local control

\section{Introduction}

The therapeutic intent in locally advanced non-small-cell lung cancer (NSCLC) is curative, and radiation therapy (RT) is a cornerstone of treatment. RT is most commonly combined with simultaneous platinum-based chemotherapy, as a concurrent approach has been shown to be su-

Frederick Mantel, MD

mantel_f@ukw.de

1 Department of Radiation Oncology, University of Würzburg, Josef-Schneider-Str. 11, 97080 Würzburg, Germany

2 Xcare Practice for Radiation Therapy, Saarlouis, Germany perior to a sequential approach with regard to local control (LC) and overall survival (OS) [1]. However, the curative intention of chemoradiotherapy (CRT) remains challenging. Progression-free survival (PFS) after 1 and 2 years has been reported to be approximately $40-50 \%$ and $20-30 \%$, respectively. The addition of consolidation chemotherapy does not prolong PFS or OS [2]. By contrast, consolidation immunotherapy by the anti-programmed death ligand 1 antibody durvalumab resulted in significantly longer progression-free and overall survival than placebo and represents the current standard of care for patients without progressive disease after CRT [3, 4]. While most patients suffer from distant progression after CRT, 30-40\% of patients experience local failure after 2 years [5]. Intensity-modulated 
radiation therapy (IMRT) combined with image-guided radiation therapy (IGRT) enables the use of moderate hypofractionation to the gross tumor volume (GTV) combined with elective nodal irradiation and possibly reduces exposure of uninvolved lung areas. Here, we report on a two-center experience of chemoradiotherapy for locally advanced NSCLC using simultaneous-integrated boost IMRT (SIBIMRT).

\section{Methods}

\section{Study patients}

We performed a retrospective analysis of 138 patients treated from 2010-2018 for locally advanced and in part oligometastatic NSCLC at the University of Würzburg (institution 1) and the Xcare practice for Radiation Therapy, Saarlouis (institution 2) using a nearly identical treatment strategy. All patients gave informed consent and we obtained approval from the ethics committee for this study. In all, 133 patients (96.4\%) underwent positron emission tomography (PET) staging. By default, clinically suspect mediastinal or supraclavicular lymph nodes were biopsy-confirmed by endobronchial ultrasound (EBUS) or mediastinoscopy, unless obvious clinical signs of lymph node metastases (e.g. mediastinal lymph node bulk) were present. All patients with oligometastatic disease were treated in institution 1. Oligometastatic disease included patients with synchronous pleural nodules, contralateral pulmonary lesions or a maximum of two synchronous extrathoracic metastases including brain, liver, bone, adrenal gland and soft tissue. Oligometastatic lesions were treated by resection and/or dose intensified radiation therapy.

As the 8th edition of the UICC (Union for International Cancer Control) TNM classification was released in the beginning of 2017, we performed a retrospective verification of the UICC TNM tumor classification and a reclassification according to the 7th and 8th edition for all patients, respectively.

\section{Treatment planning and radiotherapy}

Patients were treated with concurrent chemoradiotherapy using a SIB-IMRT technique with image-guidance by linacintegrated cone-beam computed tomography (CBCT). In institution 1 daily CBCT was acquired for the first five fractions for generation of a mean value for set-up error and then every third fraction in case detected set-up errors did not exceed $3 \mathrm{~mm}$; otherwise a CBCT was acquired prior to the subsequent fraction. In case a correction of rotational errors was reasonable, daily CBCT was performed. The decision for correction of rotational errors was made at the discretion of the treating radiation oncologist. Institution 2 used IGRT with daily CBCT for all cases. Treatment planning computed tomography (CT) with a slice-thickness of $3 \mathrm{~mm}$ was acquired in mid-ventilation and supine position with elevated arms in a vacuum mattress or another individually adjustable immobilization device. An additional 4Dplanning-CT was performed for all patients and the end-exhalation and end-inhalation phase were reconstructed and used as secondary image files in the planning software for consideration of breathing-related tumor motion in institution 1, whereas in institution 2 4D-planning-CTs were predominantly used for tumors located in the lower lobes with expected higher mobility. Pinnacle (Philips Radiation Oncology Systems, Milpitas, CA, USA) was used for treatment planning for an Elekta Synergy/Versa HD platform equipped with Agility Head/MLC (Elekta Oncology Systems, Crawley, UK). The gross tumor volume (GTV) included the macroscopic primary tumor and macroscopic lymph node metastases. Lymph nodes were considered involved when enlarged (short axis in CT $>1 \mathrm{~cm}$ ), showed an elevated standardized uptake value (SUV) $(\geq 2.5)$ or when tumor infiltration was proven by EBUS cytology. However, we followed a "generous" approach and usually included also lymph nodes, e.g., with borderline elevated SUV or those in close vicinity to proven metastatic nodes. F-18 fluorodeoxyglucose positron emission tomography (FDG-PET) information was used for GTV delineation in 133 patients by coregistration of the PET with the planning CT. FDGPET was acquired in the same position as the planning CT, i.e., in supine position with elevated arms. The GTV was contoured in free breathing. In case of 4D-CT acquisition the GTV was delineated additionally in the end-exhalation and end-inhalation position to generate an internal target volume (ITV) as the sum of the GTVs. No additional margin was added to the GTV or ITV to generate the clinical target volume (CTV) SIB. The elective CTV of hilus and mediastinum included the lymph node stations neighboring metastatic involved node regions. In institution 1 regions with a risk of metastatic involvement greater than $10 \%$ according to Giraud et al. [6] and the supraclavicular stations, in case of upper lobe primary tumor or mediastinal lymph node metastases in stations 1 or 2, were included. Contouring was done according to the atlas published by Chapet et al. [7]. An additional margin of $3-5 \mathrm{~mm}$ was added to the CTV SIB to generate the planning target volume (PTV) SIB. The elective target volume (PTV) included the CTV SIB and the elective CTV with an addition of $5-10 \mathrm{~mm}$. Patients received a follow-up planning-CT on a regular basis after $30 \mathrm{~Gy}$ for consideration of tumor response or re-ventilation in case of atelectatic lung regions in institution 1 , or earlier, if CBCT showed significant changes in comparison to the initial planning $\mathrm{CT}$, while daily CBCTs were used for deciding on the necessity to do a re-planning in 
institution 2. If tumor shrinkage was present, which in the discretion of the treating radiation oncologist enabled for a reduction of the radiation field, target volume and planadaption were performed accordingly to achieve better organ-at-risk (OAR) sparing. Both PTV and boost treatment dose was prescribed to the encompassing $95 \%$ isodose with a tolerance range of $\pm 2 \%$, typically with a single dose of 1.8 Gy to the PTV and 2.2 Gy to the PTV SIB in 28 fractions. Lungs, the heart, esophagus and spinal canal were contoured as OAR according to the NRG/RTOG contouring atlas [8]. Lung dose constraints were $30 \mathrm{~Gy} \mathrm{D}_{\text {mean }}$ a $\mathrm{V}_{5 \mathrm{~Gy}}$ of $90 \%$ and $10 \mathrm{~Gy} \mathrm{D}_{\text {mean, }} \mathrm{a} \mathrm{V}_{5 \mathrm{~Gy}}$ of $50 \%$ for the ipsilateral and contralateral lung, respectively. $\mathrm{D}_{\text {mean }}$ for both lungs together should not exceed $17 \mathrm{~Gy}$, but higher doses could be accepted at the discretion of the treating radiation oncologist. Heart $\mathrm{D}_{\text {mean }}$ was limited to $10 \mathrm{~Gy}$ and $\mathrm{D}_{01}$ to $35 \mathrm{~Gy}$ but target volume coverage had priority. Dose to the esophagus was limited to a $\mathrm{D}_{10}$ of $60 \mathrm{~Gy}$. For the spinal canal, $\mathrm{D}_{1 \mathrm{~cm} 3}$ was constrained to $45 \mathrm{~Gy}$. IGRT was used in every patient based on the regular acquisition of CBCT with online correction for set-up errors.

\section{Chemotherapy}

Concurrent chemotherapy consisted of cisplatin $20 \mathrm{mg} / \mathrm{m}^{2}$ body surface area (BSA), days 1-4, 29-32 and vinorelbine $15 \mathrm{mg} / \mathrm{m}^{2}$ BSA, days 1, 8, 15, 29, 36, 43. Alternatively, chemotherapy with weekly carboplatin area under the curve (AUC) 2 and paclitaxel $50 \mathrm{mg} / \mathrm{m}^{2}$ BSA was used. Paclitaxel mono weekly $60 \mathrm{mg} / \mathrm{m}^{2}$ BSA or carboplatin mono weekly AUC2 was applied in frail patients. Patients were selected for concomitant CRT on the basis of Karnofsky performance status (KPS), age, and comorbidities at the discretion of the treating radiation oncologist. Patients not eligible for chemotherapy were treated with SIB-IMRT only.

\section{Follow-up and endpoint assessment}

Time intervals were calculated from the date of RT completion. During follow-up (FU) CT scans were performed at each FU visit every 3 months in the first 2 years and every 6 months thereafter. Locoregional failure was defined using response evaluation criteria in solid tumors (RECIST) [9]. Local control was defined as freedom from tumor progression within the RT field. Regional control was defined as freedom from tumor progression at all mediastinal sites outside the RT field and all pulmonary sites adjacent to the primary tumor but outside the RT field. In some cases PET was performed and local failure was defined in consultation with the specialist of nuclear medicine. Radiological FU was missing for 9 patients due to early death during or after treatment and for 1 patient who was lost to FU before the first planned radiological assessment. Acute and late radiation-induced toxicity was scored by using common terminology criteria for adverse events (CTCAE) version 4.0. Acute toxicities were assessed weekly during RT and 6 weeks afterwards. Late toxicities were recorded every 3 months in the first year and every 6 months thereafter.

\section{Statistical analysis}

SPSS version 25 (SPSS, Inc.) was used for statistical analyses. Kaplan-Meier analyses were performed for local tumor control (LC), progression-free survival (PFS) and overall survival (OS) for the entire patient cohort and separately for the subgroup of patients with UICC stage III treated with concurrent CRT. Comparisons of LC in patients receiving CRT versus RT alone and of OS and PFS in stage IV versus earlier stage patients were carried out by log-rank tests. Univariate analysis for the likelihood of death or local recurrence was performed using a logistic regression. Cutoff points were chosen for UICC stage, total dose, GTV, PTV $\mathrm{SIB}, \mathrm{PTV}, \mathrm{D}_{\text {mean }}$ lungs based on receiver operating characteristic analyses. All variables that were statistically significant or trended to significance $(p \leq 0.1)$ were included in the multivariate analysis. For multivariate analysis a logistic regression with the stepwise forward method was used. A $p$ value $\leq 0.05$ was considered statistically significant.

\section{Results}

From 2010-2018, 138 patients suffering from locally advanced and in part oligometastatic NSCLC were treated in the department of radiation oncology, University of Würzburg and the Xcare practice for Radiation Therapy, Saarlouis with an identical definitive SIB-IMRT protocol. Patient and treatment characteristics are listed in Table 1. Median age was 63 years and $68 \%$ were male. Most common histology was adenocarcinoma (58\%) and squamous cell carcinoma (38\%). According to the UICC TNM classification in its 7th edition, $91(66 \%)$ patients had UICC stage $\geq$ IIIB, among them 21 (15\%) patients with oligometastatic disease (UICC stage IV). After reclassification of all 138 patients according to the 8th edition of the UICC TNM classification, $106(76 \%)$ patients had UICC stage $\geq$ IIIB and $60(43 \%)$ had stage $\geq$ IIIC. In all, $64(46 \%)$ patients had nodal stage 3 (N3). Fourteen (10\%) patients were classified as stage IVA and $7(5 \%)$ patients as stage IVB, respectively. A total of $133(96.4 \%)$ patients underwent diagnostic PET staging. Furthermore, $124(90 \%)$ patients received concurrent chemotherapy predominantly consisting of a platinumbased doublet. Fourteen $(10 \%)$ patients were physically unfit for chemotherapy treatment and were treated with SIB-IMRT only. Median SIB-IMRT boost and elective PTV total dose was 61.6 and $50.4 \mathrm{~Gy}$, respectively, in me- 
Table 1 Patient and treatment characteristics

\begin{tabular}{|c|c|c|}
\hline Parameter & & \\
\hline Age, median (range), years & 63 & $(37-89)$ \\
\hline \multicolumn{3}{|l|}{ Sex, No. $(\%)$} \\
\hline Female & 44 & $(32)$ \\
\hline Male & 94 & $(68)$ \\
\hline Karnofsky performance status, median (range), \% & 90 & $(50-100)$ \\
\hline Body mass index, median (range), $\mathrm{kg} / \mathrm{m}^{2}$ & 25 & $(16-46)$ \\
\hline$F E V_{l}$ median (range), liter & 2.1 & $(0.6-4.2)$ \\
\hline$F E V_{l} \%$ of target, median (range) & 74 & $(18-127)$ \\
\hline \multicolumn{3}{|l|}{ Histology, No. (\%) } \\
\hline Adenocarcinoma & 80 & $(58)$ \\
\hline Squamous cell carcinoma & 53 & $(38)$ \\
\hline Large-cell neuroendocrine carcinoma & 4 & (3) \\
\hline Adenocystic carcinoma & 1 & (1) \\
\hline \multicolumn{3}{|l|}{ Grading, No. (\%) } \\
\hline G1 & 1 & (1) \\
\hline G2 & 33 & $(33)$ \\
\hline G3 & 64 & (64) \\
\hline G4 & 2 & $(2)$ \\
\hline \multicolumn{3}{|l|}{ UICC tumor stage (7th edition), No. (\%) } \\
\hline $2 \mathrm{~A}$ & 1 & (1) \\
\hline 2B & 2 & (1) \\
\hline $3 \mathrm{~A}$ & 44 & $(32)$ \\
\hline 3B & 70 & $(51)$ \\
\hline 4 & 21 & $(15)$ \\
\hline \multicolumn{3}{|l|}{ UICC tumor stage (8th edition), No. (\%) } \\
\hline $2 \mathrm{~A}$ & 1 & (1) \\
\hline 2B & 2 & (2) \\
\hline $3 \mathrm{~A}$ & 29 & $(21)$ \\
\hline 3B & 46 & $(33)$ \\
\hline $3 \mathrm{C}$ & 39 & $(28)$ \\
\hline $4 \mathrm{~A}$ & 14 & $(10)$ \\
\hline $4 \mathrm{~B}$ & 7 & $(5)$ \\
\hline Cumulative total dose, median (range), Gy & 63.8 & $(54-71.6)$ \\
\hline Total No. of fractions, median (range) & 29 & $(25-33)$ \\
\hline \multicolumn{3}{|l|}{ Total dose of SIB-IMRT, median (range), Gy } \\
\hline Elective target volume (PTV) & 50.4 & $(41.4-57.1)$ \\
\hline Simultaneous integrated boost (SIB) & 61.6 & $(50.6-69.0)$ \\
\hline No. of fractions & 28 & $(20-32)$ \\
\hline Sequential boost, No. (\%) & 64 & (46) \\
\hline Total dose, median (range), Gy & 6.6 & $(2-20)$ \\
\hline No. of fractions & 3 & $(1-5)$ \\
\hline GTV, median (range), $\mathrm{cm}^{3}$ & 95.9 & $(14.7-891.7)$ \\
\hline SIB, median (range), $\mathrm{cm}^{3}$ & 186.6 & $(24.9-1361.3)$ \\
\hline$P T V$, median (range), $\mathrm{cm}^{3}$ & 689.1 & $(55.4-2401.9)$ \\
\hline
\end{tabular}

$F E V_{l}$ Forced expiratory volume in 1 second, UICC Union for International Cancer Control, Gy Gray, No. Number, SIB-IMRT Simultaneous integrated boost-intensity-modulated radiation therapy, PTV Planning target volume, GTV Gross tumor volume 

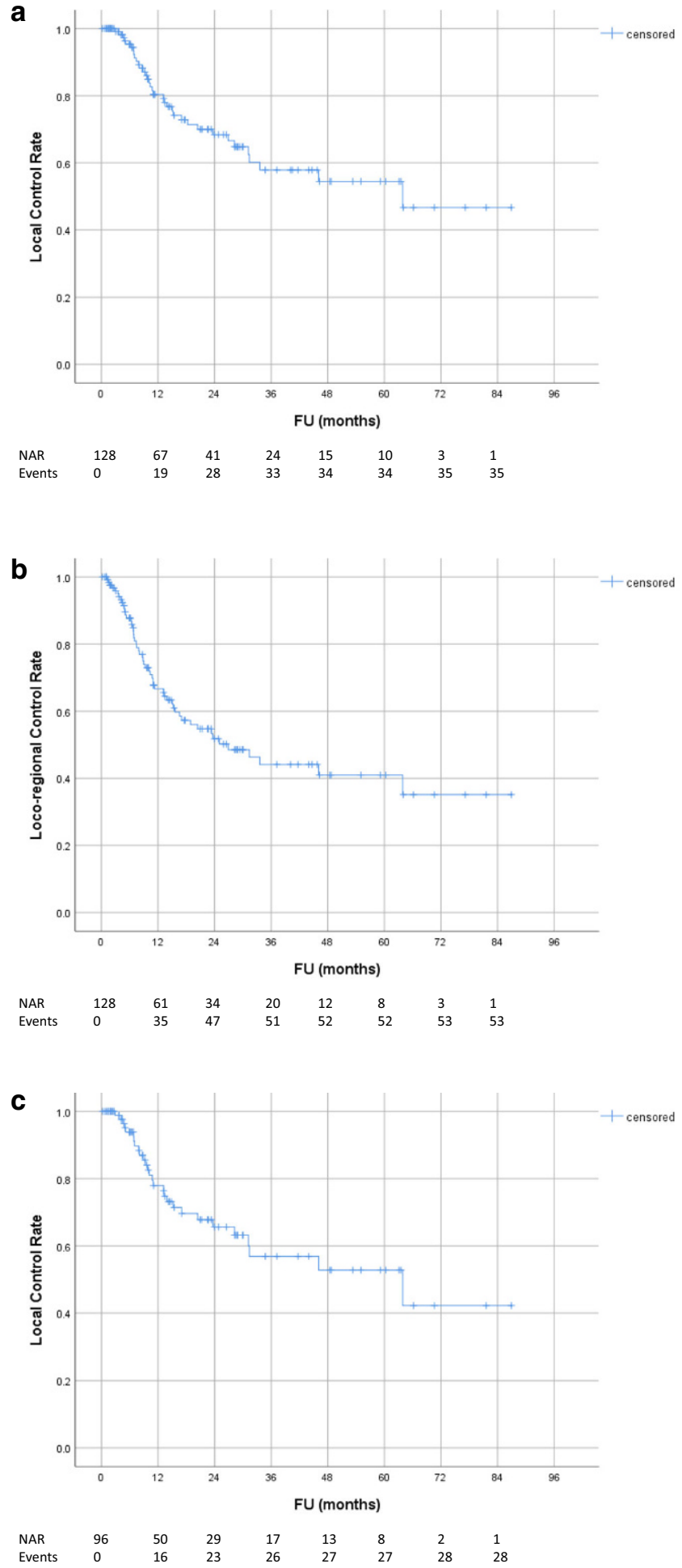

Fig. 1 Tumor control rate: a local (in-field) tumor control; b locoregional control (in- and out-field); c local (in-field) tumor control for subgroup of patients with UICC stage III treated with concurrent chemotherapy. $F U$ follow-up, NAR number of patients at risk, $C R T$ chemoradiotherapy dian 28 fractions. Sixty-nine (50\%) patients also received a plan adaption during the SIB-IMRT course due to tumor response or changes in tumor location (e.g., reventilation of atelectasis or pleural effusion). On average $27 \%$ of the PTV was included in the SIB-escalated dose region. The dose planning constraints were reliably met over a large range of PTV volumes $(55-2400 \mathrm{ml}$, median $689 \mathrm{ml})$. After completion of the SIB-IMRT course an additional sequential boost to the primary tumor was applied in 64 patients $(46 \%)$ on basis of a new planning $\mathrm{CT}$ in order to achieve further tumor regression. The sequential boost consisted of median $6.6 \mathrm{~Gy}$ in 3 fractions. This resulted in a median total dose to the primary tumor of $63.8 \mathrm{~Gy}$ (range 54.0-71.6 Gy) for all patients. Median cumulative mean lung dose was $15.6 \mathrm{~Gy}$ (range 6.2-29.5 Gy). Concurrent chemotherapy was administered per protocol in 104 patients (85\%), a dose reduction became necessary in 6 patients $(5 \%)$ and chemotherapy had to be stopped in 12 patients (10\%).

Median follow-up and radiological follow-up for all patients was 18.0 months (range 0.6-86.9) and 16.0 months (range 0.2-86.9), respectively. Local and regional tumor control rates are shown in Fig. 1. Local control rates at 1,2 and 3 years were $80.4,68.4$ and $57.8 \%$, respectively. Taking into account regional failures outside the RT field, the locoregional control rates at 1,2 and 3 years were 66.6, 51.8 and $44.1 \%$, respectively. Overall and progression-free survival rates are shown in Figs. 2 and 3: OS and PFS at 1,2 and 3 years were 72.7, 56.4, 41.0\% and 50.7, 31.4, $23.1 \%$, respectively. Median overall and progression-free survival was 30.0 months (95\% CI 23.5-36.4) and 12.1 months (95\% CI 8.2-16.0), respectively. At the 5-year FU, $71 \%$ of patients developed distant metastases. There was no statistically significant difference in OS $(p=0.30)$ or PFS $(p=0.76)$ between patients with or without oligometastasis. Moreover, local control between patients treated with CRT and patients treated with RT only did not differ significantly $(p=0.68)$. For the subgroup of UICC stage III patients that had been treated with concurrent CRT, local control rates at 1,2 and 3 years were $77.9,65.6$ and $56.9 \%$, respectively. OS and PFS at 1, 2 and 3 years were 72.5, 61.4, 44.6\% and 47.2, 27.3, 18.5\%, respectively (Figs. 1, 2 and 3). Median overall and progression-free survival in this subgroup was 30.0 months (95\% CI 25.8-34.2) and 11.0 months (95\% CI 7.5-14.5), respectively.

UICC stage (8th edition), a PTV SIB $>291 \mathrm{~cm}^{3}$, PTV and mean lung dose were statistically significant predictors of death on univariate analysis (Table 2). A PTV $\geq 600 \mathrm{~cm}^{3}$ was predictive for death on multivariate analysis. Univariate analysis revealed that UICC $>$ III B, GTV $>133 \mathrm{~cm}^{3}$, PTV SIB $>221 \mathrm{~cm}^{3}$ and Karnofsky performance status (KPS) were predictive for a local recurrence. PTV SIB $>221 \mathrm{~cm}^{3}$ and KPS were statistical significant predictors of LR on multivariate analysis (Table 3 ). 

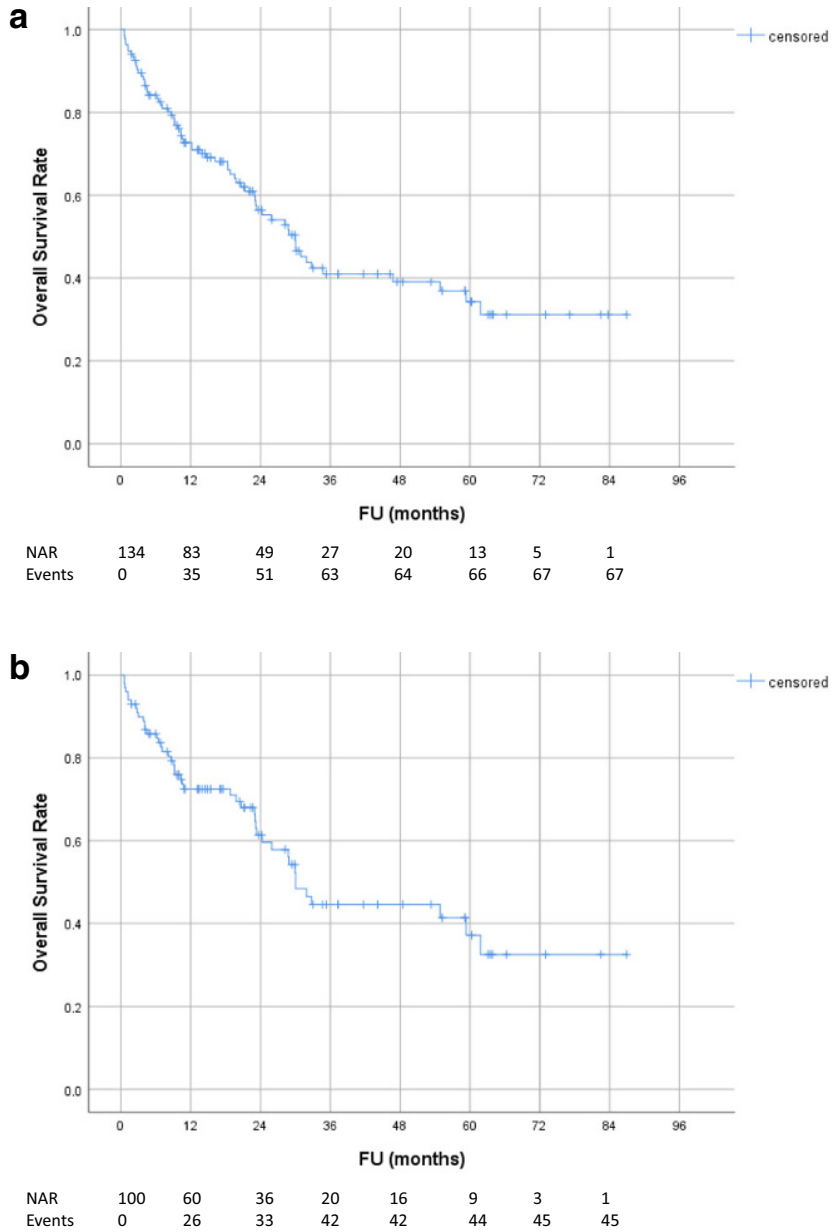

Fig. 2 Overall survival: a all patients; $\mathbf{b}$ subgroup of patients with UICC stage III treated with concurrent chemotherapy. $F U$ follow-up, $N A R$ number of patients at risk

Radiation-induced pneumonitis grade 2 and grade 3 occurred in $13(9.8 \%)$ and $3(2.3 \%)$ patients, respectively. CTCAE $\geq$ grade 3 anemia, leukopenia and thrombocytopenia was observed in $4.5,23$ and $2.8 \%$, respectively. Two patients died of tumor bleeding during treatment, and 1 patient died of progressive lymphangiosis carcinomatosa on treatment. A few weeks after completion of chemoradiotherapy, 2 patients died of a fulminant bacterial pneumonia and 1 patient after suffering from acute renal failure following a protracted infection. One patient needed pneumonectomy after CRT because of a superinfected tumor cavern. Adverse events are shown in Table 4.

\section{Discussion}

IMRT with simultaneous integrated boost was introduced into clinical practice about two decades ago. The principle of irradiating the GTV and regions at high risk of microscopic tumor spread with an increased fraction size and regions at lower risk of tumor manifestation "electively" with a lower dose permits dose escalation and higher biological efficacy of hypofractionated therapy, while sparing the surrounding tissue. This technique was first used for head and neck tumors. In lung cancer, implementation of SIB techniques is considerably more difficult due to breathing motion, changing anatomic conditions resulting from increasing or decreasing effusion, atelectasis, changes in tumor size, large differences in density of lung and solid organs or tumor. To our knowledge, the presented cohort currently documents the largest number of patients treated for locally advanced NSCLC using this technique of SIBIMRT. It shows that the SIB concept is applicable in practice with good success.

In current radio-oncological practice most commonly dose sparing to organs at risk is attempted by limiting the PTV to the primary tumor and the affected lymph node regions, an approach that became known as involved-field radiation therapy (IFRT) [10]. We recognize that there is growing evidence of IFRT not being inferior to elective nodal irradiation (ENI) with respect to locoregional failure [10-12]. However, IFRT regularly requires the addition of quite large margins to the GTV: the ESTRO guideline recommends the inclusion of the entire affected lymph node region, or at least the nodal GTV plus a $5-8 \mathrm{~mm}$ margin. To this CTV another $5-10 \mathrm{~mm}$ shall be added to generate the PTV. This volume should then be treated homogeneously with a high dose up to at least $60 \mathrm{~Gy}$ [13]. The limits of this concept are reached when large parts of the mediastinum or supraclavicular region would have to be irradiated with a high dose in multilevel N2 or N3 stages with multiple lymph node metastases. Compared to a homogeneous dose prescription, the presented SIB technique considerably lowers the volume of PTV that has to receive a dose of $>60 \mathrm{~Gy}$ in this group of patients, who accounted for a high proportion of our series: $46 \%$ of our patients had nodal stage N3. In our cohort ENI was still used. We are aware that many have abandoned this approach in favor of IFRT. However, due to the margins employed in published trials using IFRT, doses to organs at risk and side effects reported are not much different to our data [10-12]. Although the accuracy of FDG-PET with respect to TNM stage is quite high, the sensitivity to detect lymph node metastases on a per station basis is less than $60 \%$, when compared to mediastinoscopic sampling [14]. Furthermore-as in all diagnostic tests-the negative predictive value of FDG-PET decreases with the prevalence of metastases. Therefore, there is probably an increased likelihood of false-negative findings when the PET scan already detects multiple lymph node metastases. Accordingly, the ESTRO guideline also recommends a rather "generous" inclusion of borderline positive findings in PET, which somewhat blurs the border between IFR and ENI. The difference in PTV between IFRT and ENI decreases in 

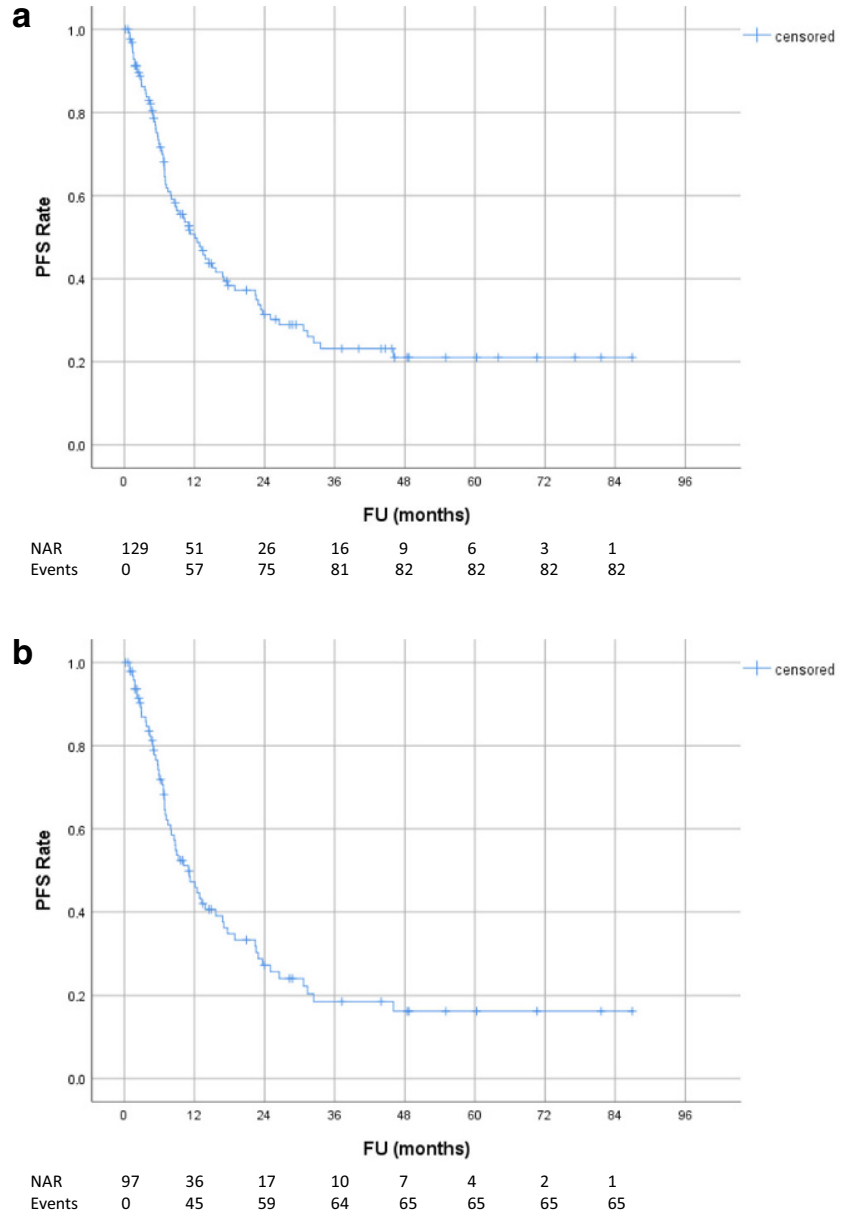

Fig. 3 Progression-free survival: a all patients; $\mathbf{b}$ subgroup of patients with UICC stage III treated with concurrent chemotherapy. PFS progression-free survival, $F U$ follow-up, NAR number of patients at risk

the presence of high stage nodal disease in the ipsi- and contralateral mediastinum. In this situation the combination of a simultaneous boost to the GTV with integrated sparing of organs at risk, both provided by SIB-IMRT may offer a relevant advantage, making a potentially curative treatment concept possible in cases where otherwise palliative radiotherapy would have been the only option. In line with this, Wang et al. found comparable outcomes for patients with locally advanced NSCLC treated by SIB-IMRT and for patients treated by conventional IMRT without SIB, although more patients with SIB-IMRT suffered from advanced N3 nodal disease [15].

SIB-IMRT with simultaneous chemotherapy led to excellent local tumor control in our patients. Though considering the observed regional failures outside the RT field and distant failures this good local control could not be transferred into convincing PFS rates, which corresponds to previous reports on CRT for locally advanced NSCLC [16]. Here, the combination of SIB-IMRT with consolidation immunotherapy with durvalumab as the present standard of care for patients showing no tumor progression contemporary after CRT hopefully will improve the outcome [3].

Obviously adoption of the new UICC classification effects relevant stage migration. Comparison to results from the literature may prove more difficult. In spite of a high proportion of UICC stage IIIC or IVA, a median survival of 30.0 months with a 5 -year overall survival of $34 \%$ was achieved using a median total dose to the primary tumor of $63.8 \mathrm{~Gy}$ in $2.2 \mathrm{~Gy}$ fractions. This is a $2 \mathrm{~Gy}$ equivalent dose (EQD2) of $64.9 \mathrm{~Gy}$ for an $\alpha / \beta$ ratio of $10 \mathrm{~Gy}$. It compares well with our preceding results using three-dimensional conformal chemoradiotherapy with a sequential boost to $66 \mathrm{~Gy}$ and no image guidance [2]. It is also in line with the findings of the RTOG 0617 trial that set the benchmark for chemoradiotherapy in NSCLC with a total dose of $60 \mathrm{~Gy}$ in $2 \mathrm{~Gy}$ fractions and found higher doses of $74 \mathrm{~Gy}$ being associated with worse local tumor control and survival $[5,17]$. The authors stated that this unexpected result was probably caused by treatment-related deaths, treatment delays and concurrent chemotherapy abortions that were more common in the $74 \mathrm{~Gy}$ than in the $60 \mathrm{~Gy}$ arm. Also protocol deviations concerning treatment planning and target volume coverage by the $95 \%$ isodose were more frequent in the high-dose group. Heart dose was shown to be significantly related to survival in that study and led to the implementation of heart dose-volume constraints in consecutive trials. Also in the meta-analysis of Ramroth et al. including 3795 patients with NSCLC from 21 randomized trials higher radiation therapy doses led to poorer survival when concurrent chemotherapy was given, but in trials without chemotherapy, higher, time-corrected biologically equivalent doses resulted in longer survival [18]. Intensely escalated dose regimens can be safely applied while not combined with full-dose chemotherapy. Belderbos et al. showed good tolerability and median overall survival rates after RT with $66 \mathrm{~Gy}$ in $2.75 \mathrm{~Gy}$ fractions combined with low-dose cisplatin [19]. In the context of fulldose concomitant chemotherapy these findings support local dose escalation only in the combination with toxicity sparing. Type and implementation of radiotherapy could therefore play an important part. Jeter et al. reported on the feasibility and outcome of IMRT with SIB for patients with stage II-IIIB NSCLC receiving standard concurrent chemotherapy. In this prospective phase I dose-escalation study the authors found a SIB dose of $72 \mathrm{~Gy}$ in 30 fractions to be the maximum tolerated dose [20]. However, their reported case numbers are very low and FU time rather short, so one should await the results of their ongoing phase II trial. $\mathrm{Li}$ et al. reported on 20 patients treated with IMRT for inoperable stage II-III NSCLC with metastatic lymph nodes [21]. They treated their patients with a total dose of 78 Gy and 60-65 Gy for the primary and the metastatic lymph nodes, respectively, prescribed simulta- 
Table 2 Risk of death

\begin{tabular}{|c|c|c|c|c|c|c|c|c|}
\hline \multirow{3}{*}{$\frac{\text { Variable }}{\text { Age }}$} & \multicolumn{4}{|c|}{ Univariate } & \multicolumn{4}{|c|}{ Multivariate } \\
\hline & \multirow{2}{*}{$\begin{array}{l}p \text { value } \\
0.13\end{array}$} & \multirow{2}{*}{$\frac{\text { Odds ratio }}{-}$} & \multicolumn{2}{|c|}{$95 \% \mathrm{CI}$} & \multirow{2}{*}{$\begin{array}{l}p \text { value } \\
-\end{array}$} & \multirow{2}{*}{$\begin{array}{l}\text { Odds ratio } \\
-\end{array}$} & \multicolumn{2}{|c|}{$95 \% \mathrm{CI}$} \\
\hline & & & - & - & & & - & - \\
\hline Sex & 0.58 & - & - & - & - & - & - & - \\
\hline UICC Stage 8th edition & 0.02 & 1.45 & 1.06 & 1.97 & 0.15 & - & - & - \\
\hline $\mathrm{UICC}>\mathrm{III} \mathrm{B}$ & 0.01 & 2.54 & 1.26 & 5.15 & 0.07 & - & - & - \\
\hline UICC IV & 0.10 & - & - & - & 0.23 & - & - & - \\
\hline Total dose & 0.16 & - & - & - & - & - & - & - \\
\hline Total dose $>68.7 \mathrm{~Gy}$ & 0.47 & - & - & - & - & - & - & - \\
\hline GTV Volume & 0.84 & - & - & - & - & - & - & - \\
\hline $\mathrm{GTV}>212 \mathrm{~cm}^{3}$ & 0.11 & - & - & - & - & - & - & - \\
\hline PTV SIB volume & 0.21 & - & - & - & - & - & - & - \\
\hline PTV SIB $>291 \mathrm{~cm}^{3}$ & 0.03 & 2.49 & 1.09 & 5.67 & 0.55 & - & - & - \\
\hline PTV & $<0.01$ & 1.00 & 1.00 & 1.00 & 0.71 & - & - & - \\
\hline $\mathrm{PTV} \geq 600 \mathrm{~cm}^{3}$ & $<0.01$ & 5.57 & 2.65 & 11.70 & $<0.01$ & 5.18 & 2.40 & 11.21 \\
\hline Lungs D Dean $(\mathrm{Gy})$ & 0.02 & 1.10 & 1.01 & 1.20 & 0.46 & - & - & - \\
\hline Lungs $\mathrm{D}_{\text {mean }}>15.44 \mathrm{~Gy}$ & $<0.01$ & 2.93 & 1.42 & 6.08 & 0.75 & - & - & - \\
\hline Simultaneous chemotherapy & 0.10 & - & - & - & 0.46 & - & - & - \\
\hline KPS & 0.10 & - & - & - & 0.27 & - & - & - \\
\hline
\end{tabular}

CI Confidence interval, UICC Union for International Cancer Control, Gy Gray, GTV Gross tumor volume, PTV Planning target volume, SIB Simultaneous integrated boost, KPS Karnofsky performance status

Table 3 Risk of local recurrence

\begin{tabular}{|c|c|c|c|c|c|c|c|c|}
\hline \multirow{3}{*}{$\frac{\text { Variable }}{\text { Age }}$} & \multicolumn{4}{|c|}{ Univariate } & \multicolumn{4}{|c|}{ Multivariate } \\
\hline & \multirow{2}{*}{$\begin{array}{l}p \text { value } \\
0.07\end{array}$} & \multirow{2}{*}{$\begin{array}{l}\text { Odds ratio } \\
-\end{array}$} & \multicolumn{2}{|c|}{$95 \% \mathrm{CI}$} & \multirow{2}{*}{$\begin{array}{l}p \text { value } \\
0.11\end{array}$} & \multirow{2}{*}{$\begin{array}{l}\text { Odds ratio } \\
-\end{array}$} & \multicolumn{2}{|c|}{$95 \% \mathrm{CI}$} \\
\hline & & & - & - & & & - & - \\
\hline Sex & 0.75 & - & - & - & - & - & - & - \\
\hline UICC Stage 8th edition & 0.07 & - & - & - & 0.09 & - & - & - \\
\hline UICC > III B & 0.04 & 2.31 & 1.05 & 5.11 & 0.09 & - & - & - \\
\hline UICC IV & 0.77 & - & - & - & - & - & - & - \\
\hline Total dose & 0.07 & - & - & - & 0.44 & - & - & - \\
\hline GTV volume & 0.68 & - & - & - & - & - & - & - \\
\hline GTV $>133 \mathrm{~cm}^{3}$ & $<0.01$ & 3.07 & 1.34 & 7.04 & 0.35 & - & - & - \\
\hline PTV SIB & 0.11 & - & - & - & - & - & - & - \\
\hline PTV SIB $>221 \mathrm{~cm}^{3}$ & $<0.01$ & 3.83 & 1.69 & 8.71 & $<0.01$ & 0.94 & 0.90 & 0.99 \\
\hline PTV volume & 0.53 & - & - & - & - & - & - & - \\
\hline PTV $>294 \mathrm{~cm}^{3}$ & 0.08 & - & - & - & 0.83 & - & - & - \\
\hline Lungs $\mathrm{D}_{\text {mean }}(\mathrm{Gy})$ & 0.07 & - & - & - & 0.69 & - & - & - \\
\hline Lungs $\mathrm{D}_{\text {mean }}>16.7 \mathrm{~Gy}$ & 0.22 & - & - & - & - & - & - & - \\
\hline Simultaneous chemotherapy & 0.72 & - & - & - & - & - & - & - \\
\hline KPS & 0.03 & 0.95 & 0.91 & 0.995 & $\mathbf{0 . 0 3}$ & 0.94 & 0.90 & 0.99 \\
\hline
\end{tabular}

CI Confidence interval, UICC Union for International Cancer Control, GTV Gross tumor volume, PTV Planning target volume, SIB Simultaneous integrated boost, Gy Gray, KPS Karnofsky performance status

neously in 26 fractions. Despite toxicity being commonly observed, the authors reported no grade $\geq 3$ acute toxicity. By contrast, fiercely escalated hypofractionation to the primary tumor or subregions of high FDG-uptake in concurrent or sequential CRT has been shown to be feasible, but associated with higher acute and late toxicities compared to conventional CRT [22]. In our study, addition of full-dose concurrent chemotherapy did not result in excessive toxicity as only moderate dose-escalation/hypofractionation in combination with SIB-IMRT and IGRT was used.

Ma et al. conducted a retrospective study on SIB-IMRT in locally advanced NSCLC [23]. In a subgroup of their patients they had combined the increase of daily applied dose to the GTV by SIB-IMRT with an additional esopha- 
Table 4 Adverse events

\begin{tabular}{|c|c|c|c|c|c|}
\hline & Grade 1 & Grade 2 & Grade 3 & Grade 4 & Grade 5 \\
\hline \multicolumn{6}{|l|}{ Acute toxicity } \\
\hline Thrombocytopenia & $32(29.4 \%)$ & $6(5.5 \%)$ & $3(2.8 \%)$ & $0(0 \%)$ & $0(0 \%)$ \\
\hline Leucopenia & $13(11.5 \%)$ & $30(26.5 \%)$ & $20(17.7 \%)$ & $6(5.3 \%)$ & $0(0 \%)$ \\
\hline Anemia & $56(50.9 \%)$ & $25(22.7 \%)$ & $5(4.5 \%)$ & $0(0 \%)$ & $0(0 \%)$ \\
\hline Renal insufficiency & $18(17.0 \%)$ & $3(2.8 \%)$ & $0(0 \%)$ & $0(0 \%)$ & $1(0.9 \%)$ \\
\hline Dysphagia & $56(40.6 \%)$ & $42(30.4 \%)$ & $15(10.9 \%)$ & $1(0.7 \%)$ & $0(0 \%)$ \\
\hline Radiation dermatitis & $49(35.5 \%)$ & $19(13.8 \%)$ & $6(4.3 \%)$ & $0(0 \%)$ & $0(0 \%)$ \\
\hline Dyspnea & $51(37 \%)$ & $12(8.7 \%)$ & $2(1.4 \%)$ & $0(0 \%)$ & $0(0 \%)$ \\
\hline Cough & $71(51.4 \%)$ & $1(0.7 \%)$ & $0(0 \%)$ & $0(0 \%)$ & $0(0 \%)$ \\
\hline Fatigue & $83(60.1 \%)$ & $10(7.2 \%)$ & $0(0 \%)$ & $0(0 \%)$ & $0(0 \%)$ \\
\hline Weight loss & $66(47.8 \%)$ & $4(2.9 \%)$ & $0(0 \%)$ & $0(0 \%)$ & $0(0 \%)$ \\
\hline Nausea & $25(18.1 \%)$ & $29(21.0 \%)$ & $1(0.7 \%)$ & - & - \\
\hline Emesis & $13(9.4 \%)$ & $9(6.5 \%)$ & $0(0 \%)$ & $0(0 \%)$ & $0(0 \%)$ \\
\hline Radiation pneumonitis & $6(4.5 \%)$ & $13(9.8 \%)$ & $3(2.3 \%)$ & $0(0 \%)$ & $0(0 \%)$ \\
\hline \multicolumn{6}{|l|}{ Late toxicity } \\
\hline Dysphagia & $14(10.8 \%)$ & $7(5.4 \%)$ & $0(0 \%)$ & $0(0 \%)$ & $0(0 \%)$ \\
\hline Skin/Soft tissue changes & $9(6.9 \%)$ & $0(0 \%)$ & $0(0 \%)$ & $0(0 \%)$ & $0(0 \%)$ \\
\hline Dyspnea & $36(27.7 \%)$ & $9(6.9 \%)$ & $0(0 \%)$ & $0(0 \%)$ & $0(0 \%)$ \\
\hline Cough & $49(37.7 \%)$ & $0(0 \%)$ & $0(0 \%)$ & $0(0 \%)$ & $0(0 \%)$ \\
\hline
\end{tabular}

gus-sparing technique resulting in a reduction of radiation esophagitis without impairing locoregional control or overall survival. Likewise, in a secondary analysis of the NRG Oncology clinical trial RTOG 0617 IMRT was associated with lower rates of severe pneumonitis and cardiac doses [24].

Several factors may have contributed to the rather favorable outcome in our cohort. Nearly all patients had PET staging before radiation therapy. PET staging reduces the number of patients with undetected multiple distant metastasized disease that have no clear benefit from treatment with chemoradiotherapy $[25,26]$. PET was also used for SIB-target volume delineation complementing 4D and 3D CTs. Supported by the accuracy of CBCT-based IGRT, metabolic active tumor lesions were reliably covered by the high-dose volume. Patients had replanning-CTs during the SIB-IMRT course and target volumes were adapted in case of relevant changes of the tumor or OAR anatomy.

In addition, SIB enabled the use of mild hypofractionation within the GTV and accelerated treatment. This might positively affect tumor control with regard to prevented tumor cell repopulation [16].

SIB reduces doses outside the target volume compared to sequential boosting. Accordingly, relatively low mean lung doses despite large PTVs were achieved in our patients. This transferred in a low rate of $12 \%$ for clinically relevant pneumonitis (mainly grade 2).

Logistic regression analysis revealed that a higher UICC stage, a higher PTV SIB, a higher PTV and mean lung dose were significantly associated with a higher risk of death on univariate analysis, and a higher PTV remained significant on multivariate analysis. We interpret these data as showing patients with locoregionally highly advanced tumor disease and multilevel nodal involvement at higher risk of death: in these patients with higher UICC stage, the multilevel nodal disease leads to higher volumes for PTV SIB and PTV and consecutively to higher mean lung doses. So the worse prognosis in these patients might probably be caused by the advanced tumor disease itself; however, causal influences by RT treatment parameters cannot be ruled out. Patients with UICC stage >III B, higher GTV, PTV SIB and lower KPS were at higher risk for local tumor recurrence on univariate analysis, and PTV SIB and KPS remained significant on multivariate analysis. Here again, advanced tumor disease associated with higher treatment volumes might itself inhere a higher risk of recurrence. A lower KPS being predictive for LR could be explained by the fact that these patients could not be treated as intensely as patients with better performance status by the addition of concurrent chemotherapy, as KPS was a major selection criteria for the application of concomitant chemotherapy. On the contrary, patients treated with RT alone showed no statistically significant difference with regard to local control in the log-rank comparison to patients treated with concomitant CRT. However, the subgroup of patients treated with RT alone was very small (10 patients) and the comparison of larger cohorts would probably favor CRT over RT. But we can conclude that SIB-IMRT without chemotherapy enables long-term local tumor control in patients not eligible for concomitant chemotherapy. 
Some patients in our study had oligometastatic disease not exceeding two extrathoracic metastases. It has been shown that patients with limited metastatic disease benefit from (chemo)radiotherapy of the primary tumor in combination with metastasis-directed local treatment with regard to PFS and OS [27-31]. In our cohort we observed a median PFS of 7.0 months and a median OS of 19.6 months for the subgroup of UICC stage 4 patients. However, this subgroup is small and heterogeneous.

There are obvious limitations to our study. The retrospective design carries the risk of underestimating toxicities. Also consecutive systemic treatments may have affected survival. Patients were not treated to the present standard of consolidative durvalumab after CRT. Local failures frequently were detected using CT and not PET-CT. The substantial decline in patients at risk most commonly due to deaths but also due to losses to FU limits the validity of tumor control and overall survival assessment. Treatmentrelated impact on patients' quality of life was not assessed. However, patients had regular follow-up and outcome/life status was updated at the time of data analysis for all patients.

Taking this into account: in this "real world" cohort of SIB-IMRT for the treatment of NSCLC from two departments' clinical routine, treatment with SIB-IMRT was very effective and tolerable.

\section{Conclusions}

Chemoradiotherapy by SIB-IMRT may result in excellent local tumor control. The overall survival appears promising regarding the high proportion of patients with UICC stage $\geq$ IIIB disease. The SIB concept resulted in low mean lung doses and minor toxicity. In the wake of consolidative immunotherapy $[3,4]$, where lung tolerance is an issue, strict IMRT-IGRT protocols with tailoring high-dose areas to the GTV should be studied in prospective trials.

Author Contribution FM, EM, PK, MZ, FE, AR, SW, SS, SH performed the data acquisition. FM, PK, BP, MF and SH performed the analyses and interpretation of the data. FM drafted the manuscript. All authors revised the manuscript critically for important intellectual content and finally approved the manuscript.

Funding Open Access funding enabled and organized by Projekt DEAL.

\section{Declarations}

Conflict of interest F. Mantel, E. Müller, P. Kleine, M. Zimmermann, F. Exner, A. Richter, S. Weick, S. Ströhle, B. Polat, S. Höcht and M. Flentje declare that they have no competing interests.
Ethical standards The Ethics Committee of the University of Würzburg approved this study (20200210 01).

Open Access This article is licensed under a Creative Commons Attribution 4.0 International License, which permits use, sharing, adaptation, distribution and reproduction in any medium or format, as long as you give appropriate credit to the original author(s) and the source, provide a link to the Creative Commons licence, and indicate if changes were made. The images or other third party material in this article are included in the article's Creative Commons licence, unless indicated otherwise in a credit line to the material. If material is not included in the article's Creative Commons licence and your intended use is not permitted by statutory regulation or exceeds the permitted use, you will need to obtain permission directly from the copyright holder. To view a copy of this licence, visit http://creativecommons.org/licenses/by/4. $0 /$.

\section{References}

1. Auperin A et al (2010) Meta-analysis of concomitant versus sequential radiochemotherapy in locally advanced non-small-cell lung cancer. J Clin Oncol 28(13):2181-2190

2. Flentje M et al (2016) GILT - a randomised phase III study of oral vinorelbine and cisplatin with concomitant radiotherapy followed by either consolidation therapy with oral vinorelbine and cisplatin or best supportive care alone in stage III non-small cell lung cancer. Strahlenther Onkol 192(4):216-222

3. Antonia SJ et al (2018) Overall survival with durvalumab after chemoradiotherapy in stage III NSCLC. N Engl J Med 379(24):2342 $-2350$

4. Antonia SJ et al (2017) Durvalumab after chemoradiotherapy in stage III non-small-cell lung cancer. N Engl J Med 377(20):1919 $-1929$

5. Bradley JD et al (2015) Standard-dose versus high-dose conformal radiotherapy with concurrent and consolidation carboplatin plus paclitaxel with or without cetuximab for patients with stage IIIA or IIIB non-small-cell lung cancer (RTOG 0617): a randomised, twoby-two factorial phase 3 study. Lancet Oncol 16(2):187-199

6. Giraud P et al (2006) Probability of mediastinal involvement in nonsmall-cell lung cancer: a statistical definition of the clinical target volume for 3-dimensional conformal radiotherapy? Int J Radiat Oncol Biol Phys 64(1):127-135

7. Chapet $\mathrm{O}$ et al (2005) CT-based definition of thoracic lymph node stations: an atlas from the University of Michigan. Int J Radiat Oncol Biol Phys 63(1):170-178

8. Kong F-M et al (2011) Consideration of dose limits for organs at risk of thoracic radiotherapy: atlas for lung, proximal bronchial tree, esophagus, spinal cord, ribs, and brachial plexus. Int J Radiat Oncol Biol Phys 81(5): 1442-1457

9. Eisenhauer EA et al (2009) New response evaluation criteria in solid tumours: revised RECIST guideline (version 1.1). Eur J Cancer 45(2):228-247

10. Rosenzweig KE et al (2007) Involved-field radiation therapy for inoperable non small-cell lung cancer. J Clin Oncol 25(35):5557-5561

11. Li R et al (2016) Involved field radiotherapy (IFRT) versus elective nodal irradiation (ENI) for locally advanced non-small cell lung cancer: a meta-analysis of incidence of elective nodal failure (ENF). Radiat Oncol 11(1):124

12. Nestle $U$ et al (2020) Imaging-based target volume reduction in chemoradiotherapy for locally advanced non-small-cell lung cancer (PET-Plan): a multicentre, open-label, randomised, controlled trial. Lancet Oncol 21(4):581-592. https://doi.org/10.1016/S14702045(20)30013-9 
13. Nestle U et al (2018) ESTRO ACROP guidelines for target volume definition in the treatment of locally advanced non-small cell lung cancer. Radiother Oncol 127(1):1-5

14. Bille A et al (2009) Preoperative intrathoracic lymph node staging in patients with non-small-cell lung cancer: accuracy of integrated positron emission tomography and computed tomography. Eur J Cardiothorac Surg 36(3):440-445

15. Wang D et al (2019) Comparison of efficacy and safety between simultaneous integrated boost intensity-modulated radiotherapy and conventional intensity-modulated radiotherapy in locally advanced non-small-cell lung cancer: a retrospective study. Radiat Oncol 14(1): 106

16. Roach MC, Bradley JD, Robinson CG (2018) Optimizing radiation dose and fractionation for the definitive treatment of locally advanced non-small cell lung cancer. J Thorac Dis 10(21):S2465S2473

17. Bradley JD et al (2020) Long-term results of NRG oncology RTOG 0617: standard- versus high-dose chemoradiotherapy with or without cetuximab for unresectable stage III non-small-cell lung cancer. J Clin Oncol 38(7):706-714. https://doi.org/10.1200/JCO.19.01162

18. Ramroth J et al (2016) Dose and fractionation in radiation therapy of curative intent for non-small cell lung cancer: meta-analysis of randomized trials. Int J Radiat Oncol Biol Phys 96(4):736-747

19. Belderbos J et al (2007) Randomised trial of sequential versus concurrent chemo-radiotherapy in patients with inoperable nonsmall cell lung cancer (EORTC 08972-22973). Eur J Cancer 43(1):114-121

20. Jeter MD et al (2018) Simultaneous integrated boost for radiation dose escalation to the gross tumor volume with intensity modulated (photon) radiation therapy or intensity modulated proton therapy and concurrent chemotherapy for stage II to III non-small cell lung cancer: a phase 1 study. Int J Radiat Oncol Biol Phys 100(3):730-737

21. Li Q-S et al (2020) Simultaneous integrated boost of intensity-modulated radiation therapy to stage II-III non-small cell lung cancer with metastatic lymph nodes. Cancer Med 9(22):8364-8372

22. van Diessen J et al (2019) The acute and late toxicity results of a randomized phase II dose-escalation trial in non-small cell lung cancer (PET-boost trial). Radiother Oncol 131:166-173
23. Ma L et al (2018) An esophagus-sparing technique to limit radiation esophagitis in locally advanced non-small cell lung cancer treated by simultaneous integrated boost intensity-modulated radiotherapy and concurrent chemotherapy. Radiat Oncol 13(1):130

24. Chun SG et al (2017) Impact of intensity-modulated radiation therapy technique for locally advanced non-small-cell lung cancer: a secondary analysis of the NRG oncology RTOG 0617 randomized clinical trial. J Clin Oncol 35(1):56-62

25. Roengvoraphoj O et al (2018) Analysis of primary tumor metabolic volume during chemoradiotherapy in locally advanced non-small cell lung cancer. Strahlenther Onkol 194(2):107-115

26. Huang YS et al (2019) Predicting tumor responses and patient survival in chemoradiotherapy-treated patients with non-smallcell lung cancer using dynamic contrast-enhanced integrated magnetic resonance-positron-emission tomography. Strahlenther Onkol 195(8):707-718

27. Gomez DR et al (2019) Local consolidative therapy vs. maintenance therapy or observation for patients with oligometastatic nonsmall-cell lung cancer: long-term results of a multi-institutional, phase II, randomized study. J Clin Oncol 37(18):1558-1565

28. Gomez DR et al (2016) Local consolidative therapy versus maintenance therapy or observation for patients with oligometastatic non-small-cell lung cancer without progression after first-line systemic therapy: a multicentre, randomised, controlled, phase 2 study. Lancet Oncol 17(12):1672-1682

29. Arrieta $O$ et al (2019) Radical consolidative treatment provides a clinical benefit and long-term survival in patients with synchronous oligometastatic non-small cell lung cancer: a phase II study. Cancer Treat Res 130:67-75

30. Iyengar $P$ et al (2018) Consolidative radiotherapy for limited metastatic non-small-cell lung cancer: a phase 2 randomized clinical trial. JAMA Oncol 4(1):e173501

31. Lievens $Y$ et al (2020) Defining oligometastatic disease from a radiation oncology perspective: An ESTRO-ASTRO consensus document. Radiother Oncol 148:157-166 